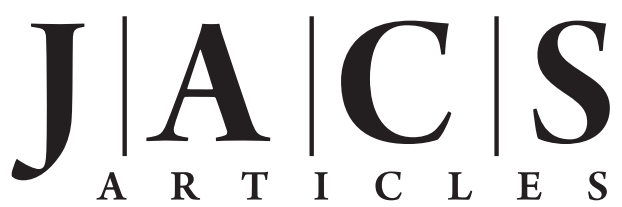

Published on Web 11/18/2010

\title{
Exciton Formation, Relaxation, and Decay in PCDTBT
}

\author{
Natalie Banerji, ${ }^{\dagger}$ Sarah Cowan, ${ }^{\dagger}$ Mario Leclerc, ${ }^{\ddagger}$ Eric Vauthey, ${ }^{\S}$ and \\ Alan J. Heeger*,† \\ Center for Polymers and Organic Solids, University of California at Santa Barbara, Santa \\ Barbara, California 93106-5090, United States, Department of Chemistry, Université Laval, \\ G1K 7P4 Quebec City, Quebec, Canada, and Department of Physical Chemistry, University of \\ Geneva, 30 Quai Ernest-Ansermet, CH-1211 Geneva 4, Switzerland
}

Received June 16, 2010; E-mail: ajhe1@ physics.ucsb.edu

\begin{abstract}
The nature and time evolution of the primary excitations in the pristine conjugated polymer, PCDTBT, are investigated by femtosecond-resolved fluorescence up-conversion spectroscopy. The extensive study includes data from PCDTBT thin film and from PCDTBT in chlorobenzene solution, compares the fluorescence dynamics for several excitation and emission wavelengths, and is complemented by polarization-sensitive measurements. The results are consistent with the photogeneration of mobile electrons and holes by interband $\pi-\pi^{\star}$ transitions, which then self-localize within about $100 \mathrm{fs}$ and evolve to a bound singlet exciton state in less than 1 ps. The excitons subsequently undergo successive migrations to lower energy localized states, which exist as a result of disorder. In parallel, there is also slow conformational relaxation of the polymer backbone. While the initial self-localization occurs faster than the time resolution of our experiment, the exciton formation, exciton migration, and conformational changes lead to a progressive relaxation of the inhomogeneously broadened emission spectrum with time constants ranging from about 500 fs to tens of picoseconds. The time scales found here for the relaxation processes in pristine PCDTBT are compared to the time scale $(<0.2 \mathrm{ps})$ previously reported for photoinduced charge transfer in phaseseparated PCDTBT:fullerene blends (Phys. Rev. B 2010, 81, 125210). We point out that exciton formation and migration in PCDTBT occur at times much longer than the ultrafast photoinduced electron transfer time in PCDTBT:fullerene blends. This disparity in time scales is not consistent with the commonly proposed idea that photoinduced charge separation occurs after diffusion of the polymer exciton to a fullerene interface. We therefore discuss alternative mechanisms that are consistent with ultrafast charge separation before localization of the primary excitation to form a bound exciton.
\end{abstract}

\section{Introduction}

In the past three decades, conjugated polymers have emerged as an important class of materials with unique properties for use in organic optoelectronics. ${ }^{1-5}$ "Plastic" solar cells represent one major application area, where the active layer is typically a blend of the semiconducting polymer with a fullerene derivative. ${ }^{6-11}$ Spontaneous phase separation leads to the formation of a

\footnotetext{
$\dagger$ University of California at Santa Barbara.

$\div$ Université Laval.

${ }^{\S}$ University of Geneva.

(1) Heeger, A. J. Chem. Soc. Rev. 2010, 39, 2354-2371.

(2) Skotheim, T. A., Reynolds, J. R., Eds. Conjugated polymers, theory, synthesis, properties, and characterization. Handbook of Conducting Polymers, 3rd ed.; CRC Press, Taylor \& Francis Group: Boca Raton, USA, 2007.

(3) Dennler, G.; Sariciftci, N. S.; Brabec, C. J. Semicond. Polym. (2nd Ed.) 2007, 2, 455-530.

(4) Malliaras, G.; Friend, R. Phys. Today 2005, 58, 53-58.

(5) Forrest, S. R. Nature 2004, 428, 911-918.

(6) Thompson, B. C.; Frechet, J. M. J. Angew. Chem., Int. Ed. 2008, 47, 58-77.

(7) Kim, J. Y.; Lee, K.; Coates, N. E.; Moses, D.; Nguyen, T.-Q.; Dante, M.; Heeger, A. J. Science 2007, 317, 222-225.

(8) Ma, W.; Yang, C.; Gong, X.; Lee, K.; Heeger, A. J. Adv. Funct. Mater. 2005, 15, 1617-1622.

(9) Brabec, C. J. Sol. Energy Mater. Sol. Cells 2004, 83, 273-292.

(10) Brabec, C. J.; Sariciftci, N. S.; Hummelen, J. C. Adv. Funct. Mater. 2001, 11, 15-26.
}

nanoscale bulk heterojunction (BHJ) material. ${ }^{12}$ Ultrafast photoinduced charge separation between the polymer donor and the fullerene acceptor is the initial step in the generation of mobile charge carriers. The mobile charge carriers can then travel to the electrodes along phase-separated fullerene and polymer networks.

To improve the performance of BHJ solar cells, synthesis has evolved toward materials with more complex molecular structures that are soluble and processable from solution, for example, functionalized poly(alkylthiophenes) and poly(phenylene vinylenes). ${ }^{1}$ Furthermore, the synthesis of alternating donor-acceptor copolymers yields small bandgap semiconductors, which enable better harvesting of the solar spectrum. Also, the low HOMO energies in these donor-acceptor copolymers lead to better stability of the pristine material and increase the open circuit voltage $\left(V_{\mathrm{OC}}\right)$ in BHJ solar cells. ${ }^{13-21}$ Power conversion efficiencies (PCE) up to $8 \%$ have been reported for solar cells comprising blends of such polymers. ${ }^{22}$ We focus on pristine PCDTBT, a copolymer within the poly(2,7-carbazole) family synthesized by Leclerc and co-workers; the molecular

(11) Shaheen, S. E.; Brabec, C. J.; Sariciftci, N. S.; Padinger, F.; Fromherz, T.; Hummelen, J. C. Appl. Phys. Lett. 2001, 78, 841-843.

(12) Yu, G.; Gao, J.; Hummelen, J. C.; Wudl, F.; Heeger, A. J. Science 1995, 270, 1789-1791. 


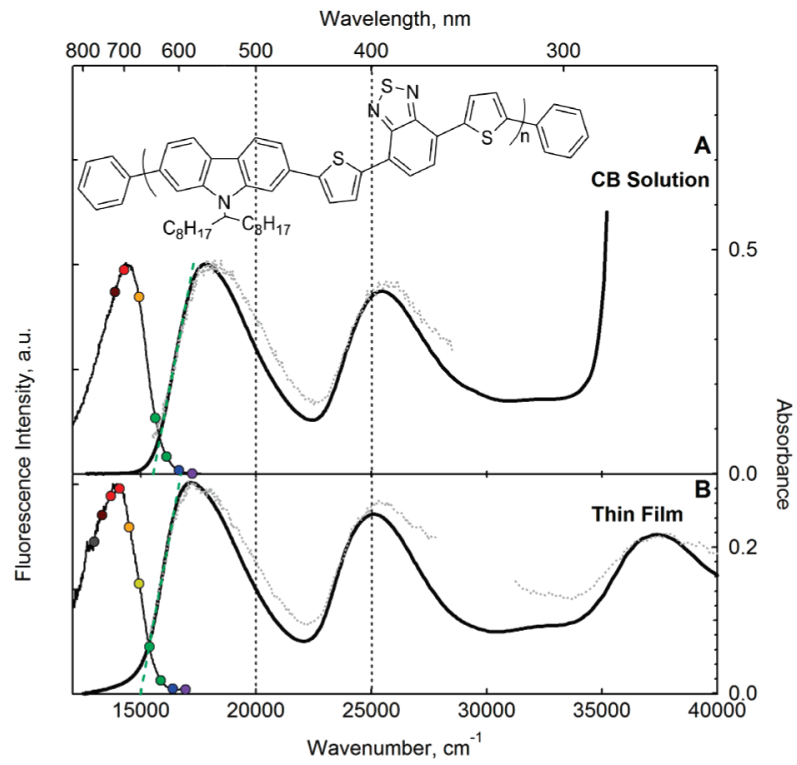

Figure 1. Steady-state spectra of PCDTBT in chlorobenzene solution (A) and as a thin film (B). The absorption spectra (thick black lines), the fluorescence excitation spectra probed near the emission maximum (dotted gray lines), and the fluorescence emission spectra with $500 \mathrm{~nm}$ excitation (thin black lines) are shown. The colored dots on the latter represent the emission wavelengths probed during the fluorescence up-conversion measurements, where excitation was achieved at 400 or $500 \mathrm{~nm}$ (vertical dashed line). The dashed green lines identify the interband absorption edge from the absorption spectrum. The molecular structure of the polymer is shown in the inset.

structure is shown in the inset of Figure $1 .{ }^{23-25}$ For PCDTBT: $\mathrm{PC}_{70} \mathrm{BM}$, photovoltaic cells with PCEs beyond 6\%, internal quantum efficiencies (IQEs) approaching 100\% have been demonstrated. ${ }^{26}$

We report here the ultrafast temporal evolution of the emission spectrum of pristine PCDTBT following light absorption, as measured by femtosecond-resolved fluorescence upconversion (FU). Data are presented for spin-cast polymer thin

(13) Liang, Y. Y.; Wu, Y.; Feng, D. Q.; Tsai, S. T.; Son, H. J.; Li, G.; Yu, L. P. J. Am. Chem. Soc. 2009, 131, 56-57.

(14) Liang, Y. Y.; Feng, D. Q.; Wu, Y.; Tsai, S. T.; Li, G.; Ray, C.; Yu, L. P. J. Am. Chem. Soc. 2009, 131, 7792-7799.

(15) Hou, J. H.; Chen, H. Y.; Zhang, S. Q.; Chen, R. I.; Yang, Y.; Wu, Y.; Li, G. J. Am. Chem. Soc. 2009, 131, 15586-15587.

(16) Peet, J.; Kim, J. Y.; Coates, N. E.; Ma, W. L.; Moses, D.; Heeger, A. J.; Bazan, G. C. Nat. Mater. 2007, 6, 497-500.

(17) Bundgaard, E.; Krebs, F. C. Sol. Energy Mater. Sol. Cells 2007, 91, 954-985.

(18) Scharber, M. C.; Muehlbacher, D.; Koppe, M.; Denk, P.; Waldauf, C.; Heeger, A. J.; Brabec, C. J. Adv. Mater. 2006, 18, 789-794.

(19) Muehlbacher, D.; Scharber, M.; Morana, M.; Zhu, Z.; Waller, D.; Gaudiana, R.; Brabec, C. Adv. Mater. 2006, 18, 2884-2889.

(20) Wang, X. J.; Perzon, E.; Oswald, F.; Langa, F.; Admassie, S.; Andersson, M. R.; Inganas, O. Adv. Funct. Mater. 2005, 15, 16651670.

(21) Zhou, Q. M.; Hou, Q.; Zheng, L. P.; Deng, X. Y.; Yu, G.; Cao, Y. Appl. Phys. Lett. 2004, 84, 1653-1655.

(22) Chen, H. Y.; Hou, J. H.; Zhang, S. Q.; Liang, Y. Y.; Yang, G. W.; Yang, Y.; Yu, L. P.; Wu, Y.; Li, G. Nat. Photonics 2009, 3, 649653.

(23) Blouin, N.; Michaud, A.; Gendron, D.; Wakim, S.; Blair, E.; NeaguPlesu, R.; Belletete, M.; Durocher, G.; Tao, Y.; Leclerc, M. J. Am Chem. Soc. 2008, 130, 732-742.

(24) Blouin, N.; Michaud, A.; Leclerc, M. Adv. Mater. 2007, 19, 2295 2300

(25) Blouin, N.; Leclerc, M. Acc. Chem. Res. 2008, 41, 1110-1119.

(26) Park, S. H.; Roy, A.; Beaupre, S.; Cho, S.; Coates, N.; Moon, J. S.; Moses, D.; Leclerc, M.; Lee, K.; Heeger, A. J. Nat. Photonics 2009, 3, 297-302. films and for PCDTBT in solution in chlorobenzene. For each, photoexcitations in the first and second absorption bands of the polymer are compared, and fluorescence polarization measurements are used to supplement the data.

Time-resolved fluorescence measurements in the pristine semiconducting polymer are of interest as an approach to study the nature and time evolution of the primary excitations. Because the photoinduced charge transfer in the PCDTBT:PC ${ }_{70} \mathrm{BM}$ blends occurs in less than $200 \mathrm{fs},{ }^{27}$ we are particularly interested in the initial relaxation processes occurring directly after the absorption of light. There exist ultrafast spectroscopic studies of new generation semiconducting polymers, such as PBTTT, ${ }^{28}$ PCPDTBT, ${ }^{29}$ or APFO3. ${ }^{30-34}$ However, detailed femtosecond investigation of the wavelength-dependent fluorescence dynamics, as described here, has not been reported for this class of materials. The nature and evolution of the primary excitations in more classic polymers, for example, PPV, ${ }^{35-46}$ or MEH-PPV, ${ }^{47-60}$ was explored by a variety of methods including FU. Still, despite

(27) Tong, M. H.; Coates, N. E.; Moses, D.; Heeger, A. J.; Beaupre, S.; Leclerc, M. Phys. Rev. B 2010, 81, 125210/1-125210/6.

(28) Hwang, I.-W.; Kim, J. Y.; Cho, S.; Yuen, J.; Coates, N.; Lee, K.; Heeney, M.; McCulloch, I.; Moses, D.; Heeger, A. J. J. Phys. Chem. C 2008, 112, 7853-7857.

(29) Hwang, I.-W.; Soci, C.; Moses, D.; Zhu, Z.; Waller, D.; Gaudiana, R.; Brabec, C. J.; Heeger, A. J. Adv. Mater. 2007, 19, 2307-2312.

(30) De, S.; Kesti, T.; Maiti, M.; Zhang, F.; Inganaes, O.; Yartsev, A.; Pascher, T.; Sundstroem, V. Chem. Phys. Lett. 2008, 350, 14-22.

(31) De, S.; Pascher, T.; Maiti, M.; Jespersen, K. G.; Kesti, T.; Zhang, F.; Inganaes, O.; Yartsev, A.; Sundstroem, V. J. Am. Chem. Soc. 2007, $129,8466-8472$.

(32) Westerling, M.; Aarnio, H.; Osterbacka, R.; Stubb, H.; King, S. M.; Monkman, A. P.; Andersson, M. R.; Jespersen, K.; Kesti, T.; Yartsev, A.; Sundstrom, V. Phys. Rev. B 2007, 75, 224306/1-224306/9.

(33) Zhang, F.; Jespersen, K. G.; Bjoerstroem, C.; Svensson, M.; Andersson, M. R.; Sundstroem, V.; Magnusson, K.; Moons, E.; Yartsev, A.; Inganaes, O. Adv. Funct. Mater. 2006, 16, 667-674.

(34) Jespersen, K. G.; Zhang, F. L.; Gadisa, A.; Sundstrom, V.; Yartsev, A.; Inganas, O. Org. Electron. 2006, 7, 235-242.

(35) Sperling, J.; Milota, F.; Kauffmann, H. F. Opt. Spectrosc. 2005, 98, $729-739$.

(36) Milota, F.; Sperling, J.; Tortschanoff, A.; Szocs, V.; Kuna, L.; Kauffmann, H. F. J. Lumin. 2004, 108, 205-209.

(37) Moses, D.; Schmechel, R.; Heeger, A. J. Synth. Met. 2003, 139, 807810.

(38) Karabunarliev, S.; Bittner, E. R. J. Chem. Phys. 2003, 118, 42914296.

(39) Sperling, J.; Milota, F.; Tortschanoff, A.; Warmuth, C.; Mollay, B.; Bassler, H.; Kauffmann, H. F. J. Chem. Phys. 2002, 117, 1087710887.

(40) Moses, D.; Wang, J.; Heeger, A. J.; Kirova, N.; Brazovski, S. Proc. Natl. Acad. Sci. U.S.A. 2001, 98, 13496-13500.

(41) Miranda, P. B.; Moses, D.; Heeger, A. J. Synth. Met. 2001, 119, 619620.

(42) Miranda, P. B.; Moses, D.; Heeger, A. J. Phys. Rev. B 2001, 6408, 081201/1-081201/4.

(43) Kennedy, S. P.; Garro, N.; Phillips, R. T. Phys. Rev. B 2001, 6411, $115206 / 1-115206 / 7$

(44) Warmuth, C.; Tortschanoff, A.; Brunner, K.; Mollay, B.; Kauffmann, H. F. J. Lumin. 1998, 76-7, 498-501.

(45) Kersting, R.; Mollay, B.; Rusch, M.; Wenisch, J.; Leising, G.; Kauffmann, H. F. J. Chem. Phys. 1997, 106, 2850-2864.

(46) Kersting, R.; Lemmer, U.; Mahrt, R. F.; Leo, K.; Kurz, H.; Bassler, H.; Gobel, E. O. Phys. Rev. Lett. 1993, 70, 3820-3823.

(47) Sperling, J.; Nemeth, A.; Baum, P.; Sanda, F.; Riedle, E.; Kauffmann, H. F.; Mukamel, S.; Milota, F. Chem. Phys. 2008, 349, 244-249.

(48) Lewis, A. J.; Ruseckas, A.; Gaudin, O. P. M.; Webster, G. R.; Burn, P. L.; Samuel, I. D. W. Org. Electron. 2006, 7, 452-456.

(49) Yang, X. J.; Dykstra, T. E.; Scholes, G. D. Phys. Rev. B 2005, 71, 045203/1-045203/15.

(50) Schindler, F.; Lupton, J. M. ChemPhysChem 2005, 6, 926-934.

(51) Ruseckas, A.; Wood, P.; Samuel, I. D. W.; Webster, G. R.; Mitchell, W. J.; Burn, P. L.; Sundstrom, V. Phys. Rev. B 2005, 72, 115214/1$115214 / 5$.

(52) Dykstra, T. E.; Kovalevskij, V.; Yang, X. J.; Scholes, G. D. Chem. Phys. 2005, 318, 21-32. 
their importance and relevance to the performance of BHJ solar cells, the early events following light absorption in pristine semiconducting polymers are not entirely understood. ${ }^{61-64}$ Understanding the evolution of the primary photoexcitations over time prior to the formation of bound excitons is of particular importance to the mechanism of operation of bulk heterojunction solar cells.

\section{Experimental Section}

2.1. Samples. The PCDTBT polymer (poly[ $N$ - $9^{\prime \prime}$-hepta-decanyl2,7-carbazole-alt-5,5-(4', $7^{\prime}$-di-2-thienyl-2', $1^{\prime}, 3^{\prime}$-benzothiadiazole)) was synthesized by Konarka Technologies $\left(M_{\mathrm{n}}=39000, M_{\mathrm{w}}=\right.$ 104000 , PDI $=2.7$ ) according to the method previously described ${ }^{23,24}$ For thin film fabrication, a circular UV-transparent and fluorescent-free fused quartz substrate (Saint-Gobain Spectrosil 2000 ) of about $3.8 \mathrm{~cm}$ diameter was used. It was first cleaned in hot piranha solution $\left(\mathrm{H}_{2} \mathrm{SO}_{4}(96 \%): \mathrm{H}_{2} \mathrm{O}_{2}(30 \%)\right.$, 2:1 volume ratio), then thoroughly rinsed and scrubbed in deionized water and ethanol, blow-dried in a $\mathrm{N}_{2}$ stream, and finally dried in an oven at $150{ }^{\circ} \mathrm{C}$ for several hours. After surface treatment in UV ozone for $1 \mathrm{~h}$, a PCDTBT thin film was spin-cast on the substrate at $4000 \mathrm{rpm}$ for $40 \mathrm{~s}$ in a nitrogen glovebox. The solution from which the film was cast was prepared in chlorobenzene (CB, Sigma-Aldrich, anhydrous, $99.8 \%$ ) at a concentration of $5.3 \mathrm{mg} / \mathrm{mL}$ and stirred overnight at $55{ }^{\circ} \mathrm{C}$ for dissolution. After the thin film was annealed on a hot plate at $60^{\circ} \mathrm{C}$ for $1 \mathrm{~h}$ and allowed to rest at room temperature for $5 \mathrm{~h}$, it was sealed (from oxygen and humidity) in a nitrogen glovebox. This encapsulation was achieved by scratching off the PCDTBT film on the edges of the substrate, applying an epoxy resin (DELO-KATIOBOND LP655) there, and then sticking a second quartz disk on top of the film. The epoxy was UV-cured at $365 \mathrm{~nm}$ for $30 \mathrm{~min}$. During this process, the polymer thin film was protected from photodamage with aluminum foil. The optical density of the solid sample was measured to be 0.24 at $400 \mathrm{~nm}$ and 0.14 at $500 \mathrm{~nm}$. The film thickness was $29 \pm 3 \mathrm{~nm}$, as determined by a surface profiler (Dektak 8 , Veeco). The steadystate absorption and fluorescence spectra of the encapsulated film revealed no sign of polymer degradation over a period of 1 month, even with the FU measurements being done in air.

For solution measurements, PCDTBT was dissolved in chlorobenzene (Acros, Extra Dry, AcroSeal, 99.8\%), which had previously been degassed by argon bubbling for $15 \mathrm{~min}$. The solution was stirred at room temperature overnight; any undissolved solid PCDTBT was then removed by filtration on cotton wool, and

(53) Burlakov, V. M.; Kawata, K.; Assender, H. E.; Briggs, G. A. D.; Ruseckas, A.; Samuel, I. D. W. Phys. Rev. B 2005, 72, 075206/1$075206 / 5$

(54) Martini, I. B.; Smith, A. D.; Schwartz, B. J. Phys. Rev. B 2004, 69 , 035204/1-035204/12.

(55) Moses, D.; Dogariu, A.; Heeger, A. J. Phys. Rev. B 2000, 61, 93739379.

(56) Dogariu, A.; Vacar, D.; Heeger, A. J. Synth. Met. 1999, 101, 202203.

(57) Hayes, G. R.; Samuel, I. D. W.; Phillips, R. T. Synth. Met. 1997, 84, 889-890.

(58) Hayes, G. R.; Samuel, I. D. W.; Phillips, R. T. Phys. Rev. B 1995, 52, 11569-11572.

(59) Hagler, T. W.; Pakbaz, K.; Heeger, A. J. Phys. Rev. B 1994, 49, 1096810975.

(60) Hagler, T. W.; Pakbaz, K.; Voss, K. F.; Heeger, A. J. Phys. Rev. B 1991, 44, 8652-8666.

(61) Scheblykin, I. G.; Yartsev, A.; Pullerits, T.; Gulbinas, V.; Sundstrom, V. J. Phys. Chem. B 2007, 111, 6303-6321.

(62) Barford, W. Electronic and Optical Properties of Conjugated Polymers; Oxford University Press: Oxford, UK, 2005.

(63) Lanzani, G.; Cerullo, G.; Polli, D.; Gambetta, A.; Zavelani-Rossi, M.; Gadermaier, C. Phys. Status Solidi A 2004, 201, 1116-1131.

(64) Sariciftci, N. S. Primary photoexcitations in conjugated polymers: Molecular exciton versus semiconductor band model; World Scientific: Singapore, 1997. the solution was finally degassed again by argon bubbling. For FU measurements, the dissolved sample was placed in a $1 \mathrm{~mm}$ cell, which consisted of two Spectrosil quartz disks separated by a Teflon spacer. The data presented in this Article were measured on samples with an optical density over $1 \mathrm{~mm}$ thickness of 0.39 at $400 \mathrm{~nm}$ (concentration of about $140 \mu \mathrm{g} / \mathrm{mL}$ ) and of 0.22 at $500 \mathrm{~nm}$ (concentration of about $100 \mu \mathrm{g} / \mathrm{mL}$ ). The solution measurements were repeated several times on different samples. Varying the PCDTBT concentration between about 60 and $160 \mu \mathrm{g} / \mathrm{mL}$, or using a solution that had been kept under argon for 2 weeks as compared to a fresh solution, had no significant effect on the shape of the steady-state spectra or on the ultrafast fluorescence dynamics.

2.2. Steady-State Measurements. Steady-state absorption spectra were measured with a Cary 50 (Varian) spectrophotometer, while fluorescence emission and excitation spectra were recorded with a Cary Eclipse (Varian) fluorimeter. All fluorescence spectra were corrected for the wavelength-dependent sensitivity of the detection. When representing the emission spectra on a wavenumber scale in $\mathrm{cm}^{-1}$, the fluorescence intensity was multiplied by the square of the wavelength $\left(\lambda^{2}\right)$ to account for the bandpass constant in wavelength imposed by the monochromator of the fluorimeter. The samples used in the steady-state measurements were the same as those used for the FU spectroscopy.

2.3. Time-Resolved Fluorescence Measurements. Emission dynamics on the femtosecond time scale was measured using the fluorescence up-conversion technique. The setup was based on a modified FOG100 system (CDP Lasers \& Scanning Systems) and has been described in detail elsewhere, ${ }^{65}$ except that a Mai Tai HP (Spectra-Physics) mode-locked Ti:sapphire laser system was now used for tunability of the excitation wavelength. In the experiments reported here, the 800 or $1000 \mathrm{~nm}$ output of the latter (100 fs pulse duration, $80 \mathrm{MHz}$ repetition rate) was frequency doubled for sample excitation at 400 or $500 \mathrm{~nm}$, respectively. The pump intensity per pulse was adjusted in the range of $10-40 \mu \mathrm{J} / \mathrm{cm}^{2}$ (with a spot diameter of $20 \mu \mathrm{m}$ ) to keep the excitation density constant at the two excitation wavelengths (of the order of $10^{14}$ photons $/ \mathrm{cm}^{3}$ in solution and $10^{18}$ photons $/ \mathrm{cm}^{3}$ in the film). The measured sample fluorescence was enhanced by sum-frequency generation with a delayed gate pulse in a nonlinear BBO crystal. Phase matching conditions were tuned to probe the fluorescence dynamics at different emission wavelengths. The up-converted signal was then dispersed in a monochromator, and its intensity was measured with a photomultiplier tube operating in the photon counting mode. The polarization of the pump beam was at magic angle $\left(54.7^{\circ}\right)$ relative to that of the gate pulses, except for polarization-sensitive measurements where it was set to $180^{\circ}$ (parallel) and $90^{\circ}$ (perpendicular). To minimize degradation, the sample cell (containing the solution or thin film) was constantly rotated during the measurement. At least two scans of the temporal dynamics in the -5 to $300 \mathrm{ps}$ (or 1000 ps) range were averaged at each emission wavelength. No significant degradation of the samples was observed between two scans or when comparing the steady-state absorption and fluorescence spectra before and after the FU measurements.

2.4. Analysis of the FU Data. For each sample, the normalized time profiles obtained by FU at various emission wavelengths were analyzed globally by adjusting a trial function to the measured data points using a nonlinear least-squares fitting procedure (Igor Pro, Wavemetrics). The dynamics could in all cases be well reproduced by the convolution of a Gaussian-shaped instrument response function (IRF) with the sum of four exponential terms. The analytical expression for this function has been described elsewhere. ${ }^{66}$ The width of the IRF was found to be around $140 \mathrm{fs}$ with thin films and around 200 fs with solution samples. The analysis allowed extraction of four global lifetimes $\left(\tau_{i}\right)$ and wavelengthdependent amplitudes (or pre-exponential factors, $a_{i, \lambda}$ ) of the

(65) Morandeira, A.; Engeli, L.; Vauthey, E. J. Phys. Chem. A 2002, 106, 4833-4837.

(66) Furstenberg, A.; Vauthey, E. J. Phys. Chem. B 2007, 111, 1261012620. 
different decay components. To relate those amplitudes to the shape of the steady-state fluorescence spectrum, they were scaled with a factor $F(\lambda)$ :

$$
F(\lambda)=\frac{S(\lambda)}{\int_{0}^{\infty} D(\lambda, t) \mathrm{d} t}=\frac{S(\lambda)}{\sum_{i} a_{i, \lambda} \tau_{i}}
$$

where $S(\lambda)$ is the corrected steady-state fluorescence intensity and $D(\lambda, t)$ is the fluorescence time profile. ${ }^{67,68}$ This scaling assumes that the steady-state emission intensity at a given wavelength is the time integral of the corresponding fluorescence time profile, and that any time constants that might have been missed in the present experiments (because they are too short or too long for the probed temporal window) do not significantly contribute to this integral. The time-resolved emission spectra were then reconstructed using the analytical expression for the time profiles at different wavelengths with the parameters (time constants and scaled amplitudes) from the fitting procedure.

For femtosecond-resolved fluorescence anisotropy measurements, FU time profiles with the polarization of the pump beam parallel $\left(I_{\|}(t)\right)$ and perpendicular $\left(I_{\perp}(t)\right)$ with respect to the gate beam were recorded. The anisotropy decay, $r(t)$, was reconstructed using the standard equation:

$$
r(t)=\frac{I_{\mathrm{II}}(t)-I_{\perp}(t)}{I_{\mathrm{II}}(t)+2 I_{\perp}(t)}
$$

This $r(t)$ was analyzed using the sum of exponential terms. When the polarization anisotropy was recorded at several emission wavelengths, this analysis was done globally. The validity of the anisotropy data was verified by comparing the magic angle time profile calculated using $I_{\|}(t)$ and $I_{\perp}(t)$ with the experimental time profile.

\section{Results}

3.1. Steady-State Spectra. As depicted in Figure 1, the absorption spectrum of PCDTBT consists of a series of broad electronic bands. Three bands can be recognized in chlorobenzene solution, peaking at 17800,25450 , and $32400 \mathrm{~cm}^{-1}(\mathrm{CB}$ absorption masks the spectrum above $34000 \mathrm{~cm}^{-1}$ ). The 32400 $\mathrm{cm}^{-1}$ transition is obviously forbidden and very weak in intensity. In thin films, the corresponding bands are red-shifted by a few hundred wavenumbers to 17200,25 050, and 32150 $\mathrm{cm}^{-1}$, but their shape is essentially the same as in solution. We were able to observe an additional band at $37320 \mathrm{~cm}^{-1}$. The bandgap is estimated from the absorption edge of the first transition (see green dashed lines in Figure 1) as equal to 15540 $\mathrm{cm}^{-1}(1.93 \mathrm{eV})$ and $15000 \mathrm{~cm}^{-1}(1.86 \mathrm{eV})$ in solution and film, respectively. For simplicity, the lowest energy absorption band will be referred to as the $S_{1}$ band and the second band as the $S_{2}$ band throughout the text.

Figure 1 shows that excitation at $20000 \mathrm{~cm}^{-1}(500 \mathrm{~nm})$, which is in the blue side of the $S_{1}$ band, leads to broad unstructured emission centered at $14400 \mathrm{~cm}^{-1}$ (solution) or $14000 \mathrm{~cm}^{-1}$ (film). The shape of the fluorescence spectrum is much narrower than the $S_{1}$ band. The Stokes shift, given by the energy difference between the emission maximum and the $\mathrm{S}_{1}$ absorption edge, is $1140 \mathrm{~cm}^{-1}(0.14 \mathrm{eV})$ in solution and 1000 $\mathrm{cm}^{-1}(0.12 \mathrm{eV})$ in the thin film. Apart from being red-shifted, the emission band in the film is also slightly broader as

(67) Horng, M. L.; Gardecki, J. A.; Papazyan, A.; Maroncelli, M. J. Phys. Chem. 1995, 99, 17311-17337.

(68) Furstenberg, A.; Vauthey, E. Photochem. Photobiol. Sci. 2005, 4, 260267.

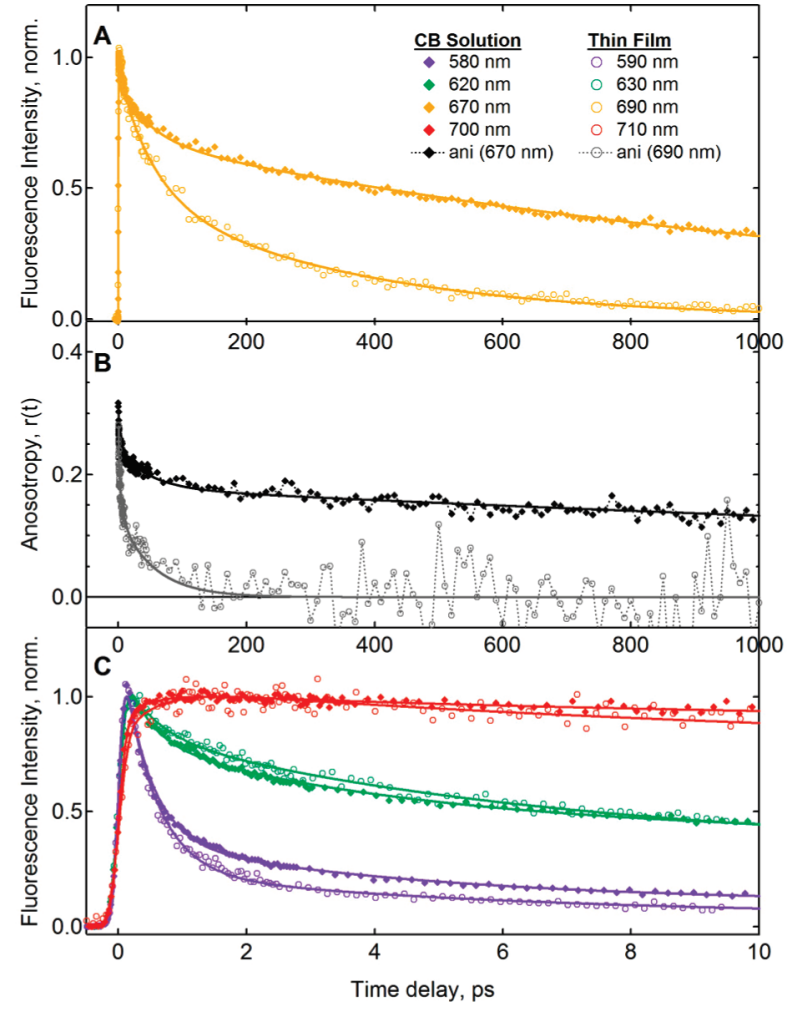

Figure 2. $(\mathrm{A}, \mathrm{C})$ Ultrafast fluorescence time profiles of PCDTBT in chlorobenzene solution $(\checkmark)$ and as a thin film $(O)$ after excitation at 500 $\mathrm{nm}$. The probed emission wavelengths are shown in the legend. (B) Time evolution of the fluorescence polarization anisotropy probed at $670 \mathrm{~nm}$ (solution, ) and $690 \mathrm{~nm}$ (film, gray O) after $500 \mathrm{~nm}$ excitation. The solid lines correspond in each case to the best multiexponential fit (see text).

compared to the emission band obtained from the solution. Finally, the excitation spectrum obtained by probing the fluorescence near the band maximum is also shown in Figure 1. In both film and solution, it resembles the absorption spectrum within the experimental uncertainty. Furthermore, the shape of the excitation spectrum was found to be independent of the probed position in the emission band (data not shown).

3.2. Fluorescence Time Profiles, $500 \mathrm{~nm}$ Excitation. In both $\mathrm{CB}$ solution and thin film, excitation at $500 \mathrm{~nm}$ occurs on the high energy side of the $S_{1}$ band. The excess energy pumped into the system relative to the bandgap is $0.52 \mathrm{eV}$ (solution) and $0.62 \mathrm{eV}$ (film). The time profiles recorded by FU near the maximum of the emission band are compared for solution and film in the top panel (A) of Figure 2. Note that the color coding corresponds to the spectral positions indicated in Figure 1. In the film, the fluorescence intensity decays almost to zero within the time window of $1 \mathrm{~ns}$, while it is much longer-lived in solution. This indicates an interchain decay pathway of the excitation in the solid state, which does not occur if the polymer is diluted in CB (at the concentration used of around $100 \mu \mathrm{g}$ / $\mathrm{mL}$, aggregation of the chains is expected to be insignificant).

Furthermore, the fluorescence dynamics in both media is clearly not single exponential and depends on the emission wavelength, especially at short time delays. This becomes more obvious in panel $\mathrm{C}$ of Figure 2, where the time profiles at different positions of the emission spectrum are shown over a temporal window of $10 \mathrm{ps}$. There is an ultrafast decay on the blue (high energy) edge of the spectrum, which becomes less pronounced when going to lower energies, until it is replaced by a rise at wavelengths higher than $670 \mathrm{~nm}$ (in film and 
Table 1. Time Constants $\left(\tau_{i}\right)$ Obtained from the Global Analysis of the Fluorescence Time Profiles Measured at Different Wavelengths

\begin{tabular}{lcccr}
\hline & $\tau_{1}(\mathrm{ps})$ & $\tau_{2}(\mathrm{ps})$ & $\tau_{3}(\mathrm{ps})$ & $\tau_{4}(\mathrm{ps})$ \\
\hline $500 \mathrm{~nm}$ exct. & & & & \\
Solution & 0.4 & 4.4 & 49.6 & 1300 \\
Film & 0.5 & 5.6 & 54.3 & 350 \\
$400 \mathrm{~nm}$ exct. & & & & \\
Solution & 0.8 & 6.9 & 73.9 & 870 \\
Film & 0.9 & 7.0 & 63.1 & 420 \\
\hline
\end{tabular}

solution). This strongly suggests an initial relaxation of the fluorescence spectrum to lower energy (red shift). When comparing similar positions of the emission band, the early fluorescence dynamics $(<10 \mathrm{ps})$ is practically identical in solution and film (Figure 2C), indicating that the initial spectral relaxation is essentially intrachain and that the interchain processes in the solid state occur on a slower time scale.

To better characterize and understand the wavelengthdependent fluorescence dynamics, they were analyzed globally (see Experimental Section for the details of the fitting procedure). All the data could be well reproduced with the sum of four exponential functions. The obtained time constants are summarized in Table 1 . The analysis using exponentials is simply a fitting procedure that provides a good analytical description of the data without imposing a specific model and it allows estimation of the time scales of the observed processes.

The result of the analysis for PCDTBT in CB solution with $500 \mathrm{~nm}$ excitation is illustrated in Figure 3; time constants of $\tau_{1}=0.4 \mathrm{ps}, \tau_{2}=4.4 \mathrm{ps}, \tau_{3}=49.6 \mathrm{ps}$, and $\tau_{4}=1.3 \mathrm{~ns}$ were found. Panel A depicts the spectra of the amplitude factors $\left(a_{i, \lambda}\right)$ associated with those time constants. Positive values correspond to decay and negative values to an increase of the emission intensity. The first two amplitude spectra, related to the shortest time constants, are characteristic of a decay of the high energy

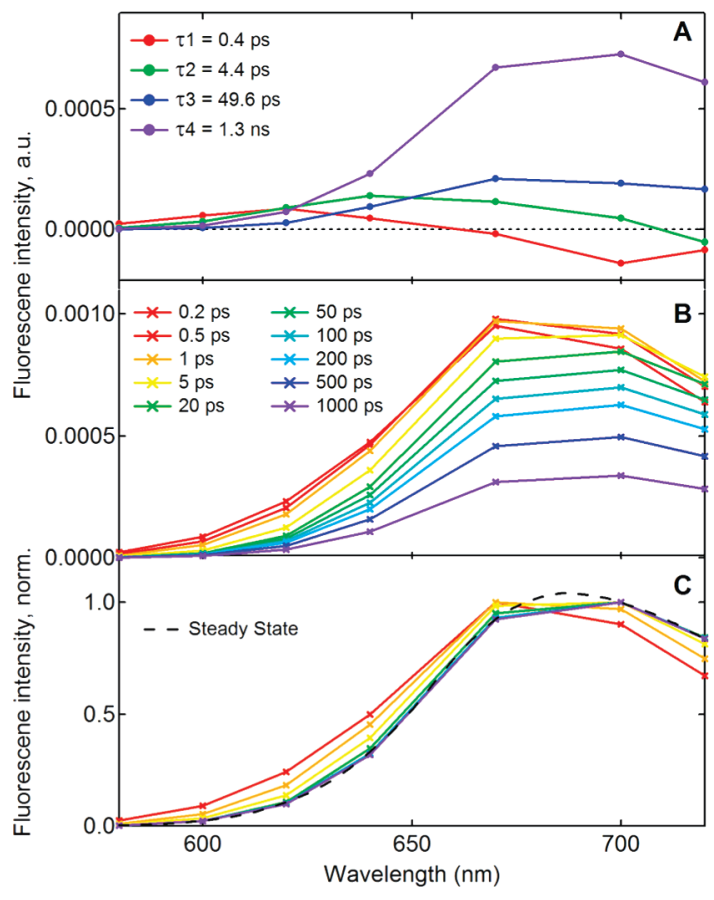

Figure 3. Outcome of the global analysis of the fluorescence time profiles of PCDTBT in chlorobenzene solution after excitation at $500 \mathrm{~nm}$. (A) Decay associated amplitude spectra. (B) Reconstructed time-resolved emission spectra. (C) Normalized reconstructed time-resolved emission spectra.

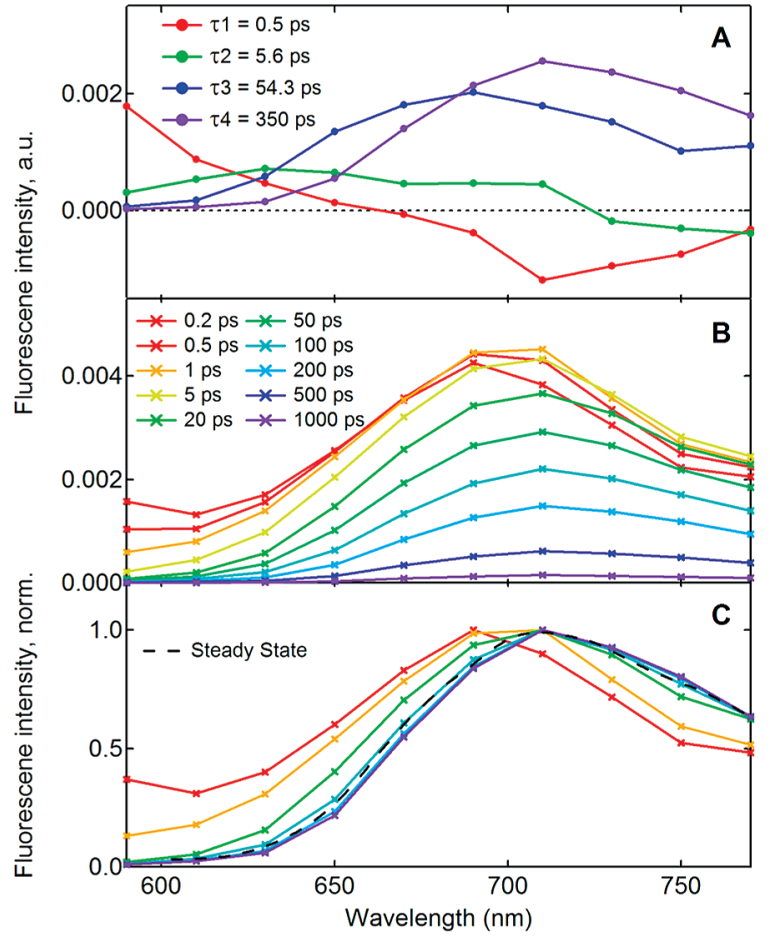

Figure 4. Global analysis of the fluorescence time profiles of PCDTBT thin film after excitation at $500 \mathrm{~nm}$. (A) Decay associated amplitude spectra. (B) Reconstructed time-resolved emission spectra. (C) Normalized reconstructed time-resolved emission spectra.

(blue) parts and a rise of the low energy (red) parts of the emission spectrum, the beginning of the rise being more redshifted for $\tau_{2}$. Those two time constants are thus ascribed to the initial spectral relaxation leading to a progressive red shift. They do not indicate a significant population decay of the excitations. On the other hand, the amplitude spectra of the slowest two time constants, $\tau_{3}$ and $\tau_{4}$, are all positive and resemble the steady-state emission spectrum. They are due essentially to decay of the excited population, although weak spectral changes continue in parallel to this decay. The temporal evolution of the fluorescence spectrum can be observed in panel B of Figure 3, which shows the reconstructed time-resolved emission spectra. Both the initial spectral relaxation and the fluorescence decay are obvious. To better visualize the spectral changes, some of the time-resolved emission spectra were normalized at maximum intensity in panel $\mathrm{C}$ of Figure 3. Apart from the red shift, the spectral dynamics includes a narrowing on the blue side and a weak broadening on the center/red side of the emission band. The spectrum has reached its final relaxed shape, which is equivalent to the steady-state emission, after 100 ps.

A similar analysis was performed on the fluorescence dynamics of PCDTBT thin film after $500 \mathrm{~nm}$ excitation. Time constants of $\tau_{1}=0.5 \mathrm{ps}, \tau_{2}=5.6 \mathrm{ps}, \tau_{3}=54.3 \mathrm{ps}$, and $\tau_{4}=$ $350 \mathrm{ps}$ were found, and the corresponding amplitude spectra are shown in Figure 4A. As in solution, the two fastest time constants are related to a progressive relaxation of the emission spectrum to lower energy. The similarity of both the time scales and the shapes of those amplitude spectra in film and solution confirm that the processes involved are essentially intrachain. However, unlike in solution, the third time constant is now clearly due to the decay of an excited population that is not yet fully relaxed (the amplitude spectrum of $\tau_{3}$ is blue-shifted as compared to steady-state emission). In the film, the relative 
Table 2. Global Time Constants $\left(\tau_{\mathrm{ri}}\right)$, Relative Amplitudes (in Parentheses), and Initial Anisotropy ( $\left.r_{0}\right)$ Obtained from the Analysis of the Fluorescence Polarization Anisotropy Measured at Different Emission Wavelengths $\left(\lambda_{\mathrm{em}}\right)$

\begin{tabular}{|c|c|c|c|c|c|c|}
\hline & $\lambda_{\mathrm{em}}(\mathrm{nm})$ & $\tau_{\mathrm{r} 1}(\mathrm{ps})$ & $\tau_{\mathrm{r} 2}(\mathrm{ps})$ & $\tau_{\mathrm{r} 3}(\mathrm{ps})$ & $\tau_{\mathrm{r} 4}(\mathrm{~ns})$ & $r_{0}$ \\
\hline \multirow{2}{*}{\multicolumn{7}{|c|}{$\begin{array}{l}500 \mathrm{~nm} \text { exct. } \\
\text { Solution }\end{array}$}} \\
\hline & & 0.4 & 4.4 & 49.6 & & \\
\hline & 600 & $(20 \%)$ & $(17 \%)$ & $(13 \%)$ & $(51 \%)$ & 0.33 \\
\hline & $670^{a}$ & $(17 \%)$ & $(6 \%)$ & $(20 \%)$ & $(57 \%)$ & 0.31 \\
\hline & 700 & $(14 \%)$ & (7\%) & (19\%) & $(61 \%)$ & 0.32 \\
\hline \multirow[t]{4}{*}{ Film } & & & 5.6 & 54.3 & & \\
\hline & 610 & $(16 \%)$ & $(37 \%)$ & $(47 \%)$ & & 0.29 \\
\hline & 690 & $(22 \%)$ & $(32 \%)$ & $(46 \%)$ & & 0.29 \\
\hline & 750 & $(37 \%)$ & $(20 \%)$ & $(44 \%)$ & & 0.33 \\
\hline \multirow{2}{*}{\multicolumn{7}{|c|}{$\begin{array}{l}400 \mathrm{~nm} \text { exct. } \\
\text { Solution }\end{array}$}} \\
\hline & & & 6.9 & 73.9 & & \\
\hline \multirow{6}{*}{ Film } & 600 & $(12 \%)$ & $(8 \%)$ & $(13 \%)$ & $(66 \%)$ & 0.28 \\
\hline & 670 & $(8 \%)$ & $(7 \%)$ & $(18 \%)$ & $(67 \%)$ & 0.27 \\
\hline & 700 & $(0 \%)$ & (9\%) & $(16 \%)$ & (74\%) & 0.24 \\
\hline & & 0.9 & 7.0 & 63.1 & & \\
\hline & 610 & $(24 \%)$ & $(31 \%)$ & $(46 \%)$ & & 0.29 \\
\hline & 690 & $(16 \%)$ & $(37 \%)$ & $(47 \%)$ & & 0.24 \\
\hline
\end{tabular}

${ }^{a}$ Values shown in italic indicate that the magic angle data could not be perfectly reproduced for this measurement.

intensity of the $\tau_{3}$ amplitudes is also more important as compared to that of $\tau_{4}$. Finally, the amplitude spectrum of $\tau_{4}$ is equal to the steady-state emission spectrum, so it is ascribed to the decay of the fully relaxed excited population. Because of interchain quenching effects, this process is much faster in the film than in solution.

In panel B of Figure 4, the time evolution of the reconstructed emission spectra for the film shows the initial rise of fluorescence intensity above $670 \mathrm{~nm}$, as well as the continuous red shift of the spectrum while it is simultaneously decaying. When the spectra of Figure 4B are normalized (Figure 4C), the spectral relaxation is even more obvious. It consists again of a red shift (by about $0.06 \mathrm{eV}$ ), a pronounced narrowing on the blue side of the emission band, and a slight broadening on the red side. In addition, at short time delays, there is a very broad underlying contribution, which seems to raise the baseline of the earliest spectra. This implies a lot of intensity at the very blue edge of the spectrum $(580-600 \mathrm{~nm})$, which was not observed in solution. Overall, the spectral changes appear slightly more important in the film than in solution, but part of this is simply because the emission spectrum is red-shifted in the film and represented on a wavelength, not energy, scale. Nevertheless, the spectral dynamics is definitely slower in the film (more than $200 \mathrm{ps}$ is required to reach the completely relaxed spectrum).

3.3. Fluorescence Anisotropy, 500 nm Excitation. The timeresolved polarization anisotropy, $r(t)$, of the fluorescence measured near the center of the emission band in $\mathrm{CB}$ solution and film, after $500 \mathrm{~nm}$ excitation, is shown in Figure 2B. Note that the top panel of the same figure represents the corresponding fluorescence time profiles measured at magic angle between the pump and gate beams. The initial anisotropy $\left(r_{0}\right)$ is 0.31 in solution and 0.29 in the film (see Table 2), which are equal within the experimental uncertainty. The value of $r_{0}$ is relatively high, given that the highest possible anisotropy, when the transition dipole moments of the excited and the emitting states are perfectly parallel, is 0.4 . The anisotropy in the film decays in a multiphasic manner and has entirely vanished after about 200 ps. The decay occurs on similar time scales as the early spectral relaxation described before, so that the first three time constants found from the global analysis of the emission dynamics were also used for the anisotropy decay (Table 2).

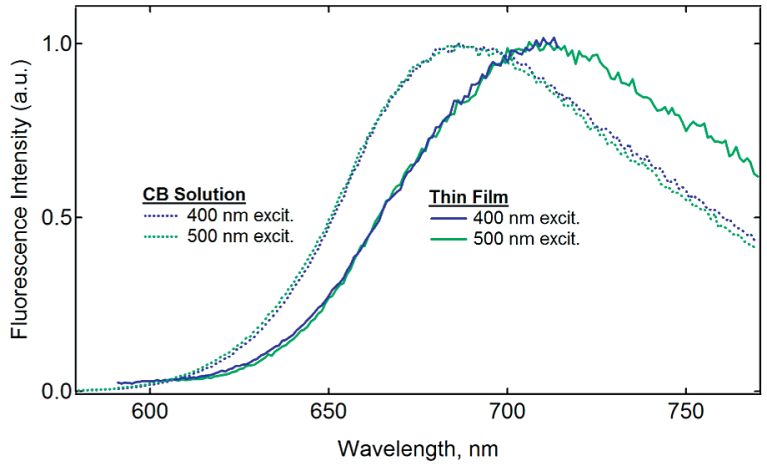

Figure 5. Steady-state fluorescence emission spectra of PCDTBT in chlorobenzene solution (dotted lines) and as a thin film (solid lines) after excitation at $400 \mathrm{~nm}$ (blue) or $500 \mathrm{~nm}$ (green).

About $22 \%$ of the anisotropy is lost with the 0.5 ps time constant, $32 \%$ with 5.6 ps, and the remaining $46 \%$ with 54.6 ps. This implies that the processes occurring during the spectral relaxation lead to a change in the orientation of the emitting transition dipole moment.

In contrast to the solid state, it is obvious in Figure 2B that only about one-half of the initial anisotropy for PCDTBT in solution is lost in parallel with the early spectral changes, while the other one-half is very long-lived. As for the film, the three time constants obtained from the analysis of the emission dynamics were used to describe the fast anisotropy decay components, while a time constant of over 3 ns was found for the slow part of the decay (Table 2). Finally, the anisotropy time profiles were measured at different positions of the emission spectrum. Data for the film with $500 \mathrm{~nm}$ excitation are shown in the Supporting Information (Figure S1). There is clearly not a strong difference between the $r(t)$ curves recorded at different wavelengths. For all samples, the anisotropy data at different wavelengths were analyzed globally with the sum of three or four exponential functions (as described above, the first three time constants were fixed to the ones found for the fluorescence dynamics). The outcome of the analysis is listed in Table 2 and confirms that the emission wavelength has only a very minor effect on the anisotropy decay. There might be a decrease in amplitude of the fastest two decay components at higher wavelengths, especially in solution, but this is difficult to confirm within the experimental uncertainty.

3.4. Fluorescence Time Profiles and Anisotropy, $400 \mathrm{~nm}$ Excitation. As shown in Figure 1, the excitation at $400 \mathrm{~nm}$ of PCDTBT in CB solution or as a thin film is at the maximum of the second $\left(\mathrm{S}_{2}\right)$ absorption band. The steady-state emission spectrum is nevertheless exactly the same, no matter where PCDTBT is initially excited (Figure 5), suggesting very fast internal conversion from the $S_{2}$ to the $S_{1}$ band and relaxation to a common emitting state.

The ultrafast fluorescence data for the thin film with $400 \mathrm{~nm}$ excitation, together with the outcome of our analysis, are presented in Figure 6. The first important comment is that all the observed fluorescence already occurs from the $S_{1}$ band (judging from the spectral position of the time-resolved emission spectra in panel $\mathrm{C}$ ). This means that internal conversion takes place within the time resolution of about $100 \mathrm{fs}$. After that, the relaxation of the spectrum continues and is completed within $200 \mathrm{ps}$, leading to a fast decay of the fluorescence time profiles measured on the blue edge of the spectrum and a rise at lower energies. The data, again analyzed with the sum of four exponential functions, and the amplitude spectra (Supporting 

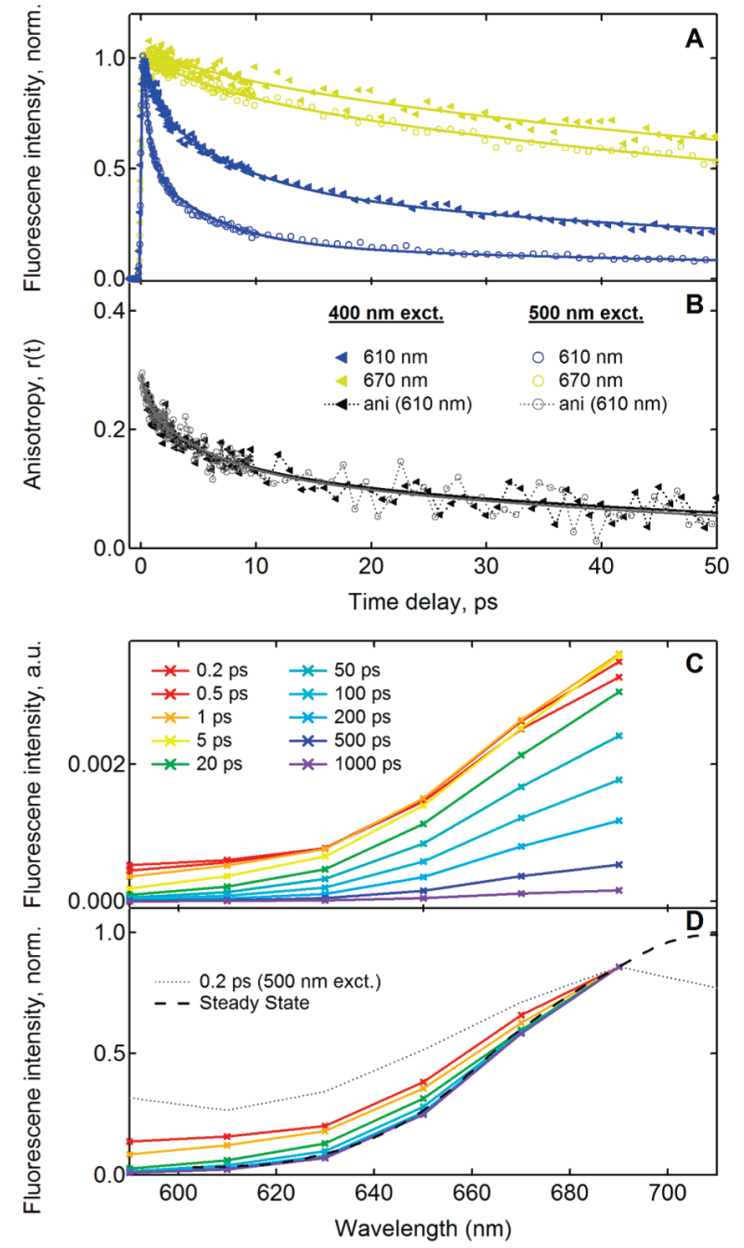

Figure 6. Ultrafast fluorescence data of PCDTBT thin film. (A) Fluorescence time profiles after excitation at $400 \mathrm{~nm}(\boldsymbol{\Delta})$ and $500 \mathrm{~nm}(\mathrm{O})$. The probed emission wavelengths are shown in the legend. (B) Time evolution of the fluorescence polarization anisotropy probed at 610 after excitation at $400 \mathrm{~nm}$ (black $\mathbf{\Delta}$ ) and $500 \mathrm{~nm}$ (gray O). For (A,B), the solid lines correspond to the best multiexponential fit. (C) Reconstructed time-resolved emission spectra after $400 \mathrm{~nm}$ excitation. (D) Normalized reconstructed time-resolved emission spectra after $400 \mathrm{~nm}$ excitation.

Information, Figure S2), are very similar to the data observed with $500 \mathrm{~nm}$ excitation. Thus, the first two time constants are related to a progressive spectral red shift, the third one is a decay of the nonrelaxed spectrum, and the slowest one is the decay of the relaxed population.

The initial anisotropy and its decay are also practically independent of excitation wavelength as well as emission wavelength (Figure 6B, Table 2), suggesting that the excited $\mathrm{S}_{1} \leftarrow \mathrm{S}_{0}$ and $\mathrm{S}_{2} \leftarrow \mathrm{S}_{0}$ transition dipole moments are parallel. As before, the first three time constants from the fluorescence time profiles were used to describe the anisotropy dynamics.

There are nevertheless also some differences between the fluorescence time profiles recorded with 400 and $500 \mathrm{~nm}$ excitation for the PCDTBT thin film. It is clear in Figure 6A that the dynamics measured at the same emission wavelengths are slower with $400 \mathrm{~nm}$ excitation, especially at the high energy (blue) end of the spectrum. This is confirmed by the slightly longer time constants for the initial spectral relaxation found with $400 \mathrm{~nm}$ excitation $\left(\tau_{1}=0.9 \mathrm{ps}, \tau_{2}=7.0 \mathrm{ps}, \tau_{3}=63.1 \mathrm{ps}\right.$; see Table 1$)$. The decay of the relaxed spectrum $\left(\tau_{4}=420 \mathrm{ps}\right)$ occurs on a similar time scale with both excitation wavelengths, considering that the experimental uncertainty becomes larger for such long time constants (delay stage misalignment, dynamics only measured up to 300 or 1000 ps). Another important difference between the excitation wavelengths is that the initial spectral rise, associated with the subpicosecond time constant, occurs at all wavelengths above $630 \mathrm{~nm}$ with $400 \mathrm{~nm}$ excitation, while it only starts above $670 \mathrm{~nm}$ with $500 \mathrm{~nm}$ excitation (Figure $6 \mathrm{C})$. The normalized time-resolved emission spectra in panel $\mathrm{D}$ of the same figure show the red shift and red-side narrowing occurring during the spectral relaxation. Note that experimental constraints with $400 \mathrm{~nm}$ excitation do not allow measurement beyond $700 \mathrm{~nm}$. Interestingly, the spectral dynamics is less pronounced than with $500 \mathrm{~nm}$ excitation (for which the spectrum at $0.2 \mathrm{ps}$ is shown as a gray dotted line for comparison). It seems that at this early time delay, the spectrum is more relaxed if initially excited at $400 \mathrm{~nm}$, and that the broad background (high baseline) is less obvious.

Finally, the fluorescence data for the polymer in CB solution with $400 \mathrm{~nm}$ excitation are shown in the Supporting Information (see Figure S3 for the time profiles and Figure S4 for the global analysis). The amplitude spectra are again quite similar to those obtained with $500 \mathrm{~nm}$ excitation and can be interpreted in the same manner. The differences observed in the film between the two excitation wavelengths also occur in solution. Thus, as seen in Figure S3A and Table 1, the time constants for the fast relaxation processes are slower with $S_{2}$ excitation $\left(\tau_{1}=0.8 \mathrm{ps}\right.$, $\tau_{2}=6.9 \mathrm{ps}, \tau_{3}=73.9 \mathrm{ps}$ ), while the decay of the relaxed spectrum is comparable. Note that the two fastest time constants obtained with $400 \mathrm{~nm}$ excitation in film and solution are identical, pointing again to intrachain processes. Concerning the reconstructed time-resolved emission spectra (Figure S4B and C), the subpicosecond spectral rise occurs above $640 \mathrm{~nm}$ with $400 \mathrm{~nm}$ excitation, while it only starts at $670 \mathrm{~nm}$ with $500 \mathrm{~nm}$ excitation. Also, the spectral changes are a little less pronounced with the higher excitation energy, but the effect is smaller than in the film. As in the film, there is no significant effect of the excitation wavelength on the initial anisotropy and its decay, no matter at which emission wavelength it is recorded (Figure S3B, Table 2).

\section{Discussion}

On the basis of experimental results obtained from highly oriented MEH-PPV chains in a polyethylene matrix, ${ }^{59,60,69,70}$ and of evidence obtained from photoconductivity studies and ultrafast investigations of the IRAV modes in PPV and MEH-PPV, ${ }^{37,40-42,55,71}$ we view conjugated polymers as quasi one-dimensional semiconductors and describe their electronic structure within an energy band picture. ${ }^{1,72}$

4.1. Steady-State Spectra. PCDTBT is characterized by a relatively large donor-acceptor repeat unit. The absorption spectrum consists of several broad bands, as commonly observed in donor-acceptor copolymers of similar chemical structure. ${ }^{32,73}$ We interpret all the intense absorption bands as allowed intrachain $\pi-\pi^{*}$ transitions yielding electrons and holes in the

(69) Hagler, T. W.; Pakbaz, K.; Heeger, A. J. Phys. Rev. B 1995, 51, 1419914206.

(70) Hagler, T. W.; Pakbaz, K.; Moulton, J.; Wudl, F.; Smith, P.; Heeger, A. J. Polym. Commun. 1991, 32, 339-342.

(71) Moses, D.; Soci, C.; Miranda, P.; Heeger, A. J. Chem. Phys. Lett. 2001, 350, 531-536.

(72) Heeger, A. J.; Sariciftci, N. S.; Namdas, E. B. Semiconducting and Metallic Polymers; Oxford University Press: Oxford, UK, 2010.

(73) Jespersen, K. G.; Beenken, W. J. D.; Zaushitsyn, Y.; Yartsev, A.; Andersson, M.; Pullerits, T.; Sundstrom, V. J. Chem. Phys. 2004, 121, 12613-12617. 
conduction and valence band, respectively. The splitting of the electronic structure into several sub- $\pi$ bands is explained by the large number of atoms in the monomer unit. The emission spectrum consists of a single unstructured band, which is not the mirror image of the first absorption band (the latter is broader). While the absorption transition is ascribed to the lowest $\pi-\pi^{*}$ interband transition, the emission occurs from a localized and relaxed exciton. Even larger differences between the absorption and emission spectral shapes are found with other polymers such as MEH-PPV or P3HT. ${ }^{1,74}$ The Stokes shift is another important feature. All relaxation processes that lower the energy of the system after photoexcitation contribute to the Stokes shift. Those include thermalization of the electrons and holes to the edge of the conduction and valence band, respectively, vibrational relaxation, localization of the excitation, exciton formation, exciton migration, and structural relaxation.

4.2. Relaxation of the Emission Spectrum Following $S_{1}$ Excitation. The time-resolved fluorescence studies were mainly carried out to characterize the initial relaxation processes in PCDTBT following light absorption. We note that, in both solution and film, about one-half of the relaxation occurs faster than the instrumental time resolution of about 100-200 fs, while the other one-half can be resolved by our experiments. For example, in the thin film following $500 \mathrm{~nm}$ excitation, the total Stokes shift is about $0.12 \mathrm{eV}$, but only $0.06 \mathrm{eV}$ of this shift can be observed, while the rest occurs within the time resolution.

We ascribe the ultrafast $(<100 \mathrm{fs})$ part of relaxation to thermalization of the mobile electrons and holes toward the band edges and to their self-localization, driven by local structural lattice distortions that are strongly coupled to the electronic states of the localized charges. ${ }^{75}$ Both processes are entangled with vibrational relaxation. ${ }^{76}$ The initial self-localization of the photoexcitation within tens of femtoseconds by structural relaxation has been independently reported by several groups. Dynamic localization of the excitation in conjugated MEH-PPV was, for example, observed by a faster decay of the polarization anisotropy in the fluorescence and transient absorption dynamics, as compared to derivatives with shorter conjugation length, ${ }^{51}$ as well as by three-pulse photon echo experiments in films and solution. ${ }^{49,52}$ Loss of coherence (electronic dephasing) within $100 \mathrm{fs}$ seen in low temperature fluorescence interferograms of PPV was also ascribed to conformational changes following the initial excitation. ${ }^{36}$ As expected, ${ }^{51,77,78}$ this self-localization in pristine PCDTBT leads to an ultrafast depolarization of the fluorescence in our experiments. Indeed, the measured initial anisotropy is only of about 0.3 in both film and solution. Before any relaxation, the absorbing and emitting transition dipole moments are parallel, and the anisotropy is 0.4 , implying an unresolved anisotropy decay component from 0.4 to 0.3 . The similar measured initial anisotropy in film and solution indicates that essentially the same processes occur and that they are of intrachain origin.

The fact that only one-half of the Stokes shift occurs faster than the time resolution indicates that the earliest fluorescence

(74) Cook, S.; Furube, A.; Katoh, R. Energy Environ. Sci. 2008, 1, 294 299.

(75) Su, W. P.; Schrieffer, J. R. Proc. Natl. Acad. Sci. U.S.A. 1980, 77, $5626-5629$.

(76) Pigliucci, A.; Duvanel, G.; Daku, L. M. L.; Vauthey, E. J. Phys. Chem. A 2007, 111, 6135-6145.

(77) Grage, M. M. L.; Zaushitsyn, Y.; Yartsev, A.; Chachisvilis, M.; Sundstrom, V.; Pullerits, T. Phys. Rev. B 2003, 67, 205207/1-205207/ 5.

(78) Grage, M. M. L.; Pullerits, T.; Ruseckas, A.; Theander, M.; Inganas, O.; Sundstrom, V. Chem. Phys. Lett. 2001, 339, 96-102. recorded after 100-200 fs does not yet originate from a relaxed exciton. Indeed, even if the FU measurements cannot resolve the fastest relaxation processes, slower spectral changes of the emission (a red shift, narrowing on the blue side and broadening on the red side) are clearly seen following $500 \mathrm{~nm}$ excitation in solution and film. They are multiphasic and can be described by at least three time constants: one of about $500 \mathrm{fs}$, one of about $5 \mathrm{ps}$, and one of several tens of picoseconds. As the different relaxation processes are complex and occur in parallel, it is impossible to assign each time constant to a specific relaxation route.

Nevertheless, the data lead to some solid conclusions. Given the large fraction of the Stokes shift occurring after about 200 fs, we conclude that the exciton is not yet entirely formed at this time delay. Therefore, the fastest subpicosecond time constant $(\sim 500 \mathrm{fs})$ is partly associated with slower components of the electron and hole relaxation to the band edges and with the formation of the singlet excitons (which involves spatial recombination and Coulomb binding of the electrons and holes after they have self-localized). In particular, the spectral rise of the lower energy parts of the emission spectrum within $1 \mathrm{ps}$ is assigned to exciton formation.

Localized states, which are present near the band edge due to disorder, ${ }^{79}$ are seen as a band tail in the steady-state absorption spectrum of PCDTBT. Once formed following photoexcitation, the singlet excitons can hop to and between localized states and change their spatial position and energy. This process has often been described within the "molecular picture" as excitation energy transfer, mainly by the Förster mechanism, between polymer segments of different conjugation lengths (chromophores, spectroscopic units). ${ }^{61}$ Cascading downenergy exciton hopping typically leads to a progressive red shift of the fluorescence spectrum, which slows in time as the number of nearby states with lower energy decreases. Time-resolved emission studies, Monte Carlo simulations, and ultrafast transient absorption polarization measurements have been carried out to investigate the phenomenon in PPV, ${ }^{35,39,43-46} \mathrm{MEH}-\mathrm{PPV}$ and derivatives, ${ }^{57,58}$ polyfluorene, ${ }^{80}$ rigid stepladder type copolymers, ${ }^{81}$ and a polythiophene polymer. ${ }^{77,78}$ Time scales ranging from subpicoseconds to hundreds of picoseconds were reported, so that we conclude that exciton hopping contributes to the three time scales $(\sim 500 \mathrm{fs}, \sim 5 \mathrm{ps}, \sim 50 \mathrm{ps})$ of the spectral relaxation observed here for PCDTBT in solution and film. The fastest one-step hops occur in $0.5-1 \mathrm{ps}$, in agreement with literature, and then cascading and decelerating energy migration leads to the observed progressive red shift of the emission spectrum.

Finally, slow conformational changes of the backbone also contribute to the spectral changes taking place within picoseconds and tens of picoseconds. ${ }^{82,83}$ Those have previously been investigated with oligomeric model systems in solvents of varying viscosity. For example, oligomeric phenylene(ethynylenes) undergo planarization in the excited state ${ }^{84}$ Further-

(79) Mott, N.; Pepper, M.; Pollitt, S.; Wallis, R. H.; Adkins, C. J. Proc. R. Soc. London, Ser. A 1975, 345, 169-205.

(80) Meskers, S. C. J.; Hubner, J.; Oestreich, M.; Bassler, H. J. Phys. Chem. $B$ 2001, 105, 9139-9149.

(81) Pina, J.; de Melo, J. S.; Burrows, H. D.; Bunnagel, T. W.; Dolfen, D.; Kudla, C. J.; Scherf, U. J. Phys. Chem. B 2009, 113, 15928-15936.

(82) Di Paolo, R. E.; de Melo, J. S.; Pina, J.; Burrows, H. D.; Morgado, J.; Macanita, A. L. ChemPhysChem 2007, 8, 2657-2664.

(83) Hintschich, S. I.; Dias, F. B.; Monkman, A. P. Phys. Rev. B 2006, 74, 045210/1-045210/10.

(84) Duvanel, G.; Grilj, J.; Schuwey, A.; Gossauer, A.; Vauthey, E. Photochem. Photobiol. Sci. 2007, 6, 956-963. 
more, in a detailed time-resolved fluorescence study of P3HT in dilute solution, both energy transfer and thermally activated torsional relaxation were used to interpret slower changes in the emission spectrum $(1-100 \mathrm{ps})$ after the initial localization of the excitation. ${ }^{85}$ These structural rearrangements are expected to be more hindered in the solid state than in solution. This explains why, in our PCDTBT data, it takes about 200 ps to reach the relaxed spectrum in the thin film, but only about 100 ps in chlorobenzene. At all emission wavelengths, there is a multiphasic decay of the polarization anisotropy, with the same time constants observed for the spectral relaxation. Depolarization is consistent with both conformational changes (the transition dipole moment reorients) and with exciton hopping to a state with a different orientation of the emitting dipole. Note that exciton hopping occurs not only to lower energy sites, but also to sites with similar energy. The isoenergetic hops can lead to depolarization, but not to any shift of the emission spectrum.

4.3. Relaxation of the Emission Spectrum Following $\mathbf{S}_{\mathbf{2}}$ Excitation. When the $S_{2}$ band of PCDTBT is excited, internal conversion to the $S_{1}$ band adds to the other relaxation processes. It is already clear from the independence of the steady-state fluorescence spectrum on the excitation wavelength and from the resemblance of the absorption and fluorescence excitation spectra that PCDTBT always relaxes to the same bound exciton state, no matter whether the photon is absorbed in the $S_{1}$ or $S_{2}$ $\pi-\pi^{*}$ transition. The FU measurements with $400 \mathrm{~nm}$ excitation were carried out to elucidate how the system gets there. They show that internal conversion (and any relaxation inside the $S_{2}$ band) occurs within the 100-200 fs instrumental time resolution, for solution and film. This is a very important result in view of our previously reported observation that mobile polarons are formed in the thin film in higher yield from the $S_{2}$ band than from the $S_{1}$ band. ${ }^{27}$ Those mobile charge carriers lead to macroscopic photocurrent in the pristine polymer film. They occur when electrons and holes from interband absorption manage to self-localize as polarons (defined as a charge coupled to a structural distortion) on separate polymer chains. We have shown here that the mobile polarons on separate chains are created extremely fast following $S_{2}$ excitation, before internal conversion to the lower energy $S_{1}$ band and before the formation of a bound exciton state.

Note that the higher yield of free polarons (which do not contribute to fluorescence) from the second absorption band implies a relative decrease in amplitude of the corresponding band in the fluorescence excitation spectrum compared to the absorption spectrum. The effect is, however, expected to be very small, given the low overall yield of macroscopic photocurrent (of the order of a few percent), as most photoexcited electrons and holes stay on the same polymer chain and geminately recombine to excitons. It can therefore not be discerned in the fluorescence excitation spectrum depicted in Figure 1. Indeed, the experimental uncertainty related to a tilt in the baseline masks any slight reduction of the spectral amplitude at high energy.

When the $S_{2}$ band is initially excited, the relaxation processes that can be resolved after internal conversion in the $S_{1}$ band are very similar to the ones observed with direct $S_{1}$ excitation. They lead to the same relaxed spectrum on the same time scale (100 ps in solution, $200 \mathrm{ps}$ in the film) and are again identical

(85) Wells, N. P.; Boudouris, B. W.; Hillmyer, M. A.; Blank, D. A. J. Phys. Chem. C 2007, 111, 15404-15414. in the two media before $10 \mathrm{ps.} \mathrm{Furthermore,} \mathrm{the} \mathrm{similar} \mathrm{initial}$ anisotropy with 400 and $500 \mathrm{~nm}$ excitation indicates that the transition dipole moments for $\mathrm{S}_{1}$ and $\mathrm{S}_{2}$ absorption are parallel, and that there is no reorientation of the emission dipole during the internal conversion. However, the fast spectral rise $(<1 \mathrm{ps})$, which we ascribe to exciton formation, starts at higher energy with $S_{2}$ excitation. This implies that the excitation is further from the band edges after internal conversion and therefore has to relax more toward the band edge. As this rise does not contribute significantly to spectral shape changes, this information is lost once the time-resolved spectra are normalized. It becomes, on the other hand, clear that the shape of the earliest shown spectrum at $200 \mathrm{fs}$ is closer to that of the relaxed spectrum with $S_{2}$ as compared to $S_{1}$ absorption. Also, the relaxation time constants are slower with excitation in the second band, which we ascribe to slower down-energy exciton hopping. We suggest that this is because different localized states, with lower energy, are populated after internal conversion. Indeed, the self-localization occurs on a very similar sub-100 fs time scale as the internal conversion, so that it might be affected and lead to a different population distribution of disordered localized states.

4.4. Comparison between Solution and Film. For the measurements in dilute chlorobenzene solution, aggregation is expected to be negligible so that the polymer chains can be considered independent from one another. Not much is, however, known about the conformation of the PCDTBT molecules in solution. Disorder due to torsion, coiling, or even folding of the backbone can be expected and leads to inhomogeneous broadening of the steady-state spectra. The slight red shift of the absorption and emission spectrum in the thin film of PCDTBT might be due to interchain interactions or to the higher refractive index in the film. Judging from the similar shape and negligible broadening of the spectra in the film as compared to solution, we conclude that interchain interactions are weak and that the morphology of the film is amorphous. Much larger spectral differences between film and solution are observed for polymers with more crystalline morphology such as P3HT. ${ }^{74}$ Furthermore, the comparable inhomogeneous broadening in film and solution points to similar disorder, indicating that the chains do not change their conformation very much upon being spin-cast from chlorobenzene solution.

In the time-resolved data, the practically identical early time constants, emission dynamics, and anisotropy indicate that the photophysics of PCDTBT in chlorobenzene solution and in the thin film cast from the same solvent are surprisingly similar during the first $10 \mathrm{ps}$ after excitation. This confirms that in both cases, the excitation is essentially intrachain and that the faster relaxation processes (self-localization, exciton formation, and first exciton migration steps) occur within the polymer chain that initially absorbed the light. The similarity also points to a comparable chain conformation in solution and in the amorphous film, as noted above based on the steady-state spectra.

Differences between the two media appear only on the tens of picosecond time scale. As interactions of polymer chains are negligible in dilute solution but present in the film, these differences can be related to interchain processes. First, there is the slower spectral relaxation in the film, which was already discussed in terms of higher hindrance of conformational changes. Second, the polarization anisotropy decays entirely in about 200 ps in the solid state, while about one-half of the initial anisotropy survives on a nanosecond time scale in solution. We ascribe exciton hopping to neighboring chains, which is slower 
than intrachain hopping, to the complete loss of anisotropy in the film. Indeed, the reorientation of the transition dipole moment is expected to be much more important by sequential migrations to disordered sites in an amorphous film. Furthermore, the significant and very long-lived anisotropy component in solution indicates a somewhat extended chain configuration, as a higher loss of polarization would occur by intrachain exciton hopping if the polymer was completely coiled up or folded. After the spectral relaxation is complete in about 100 ps, anisotropy in the isolated chain is only lost by very slow orientational diffusion of the polymer molecule.

The third difference is that the singlet exciton population decays faster in the film than in solution. Such concentration quenching effects in spin-cast films, leading to a lower fluorescence quantum yield as compared to solution, are common in conjugated polymers and are ascribed to an increase of radiationless processes. ${ }^{74,81}$ The elucidation of the precise exciton decay mechanism in PCDTBT film and solution needs further investigation, for example, by transient absorption spectroscopy. Possible decay pathways include intersystem crossing to the triplet state, dissociation at impurities, dissociation to create polaron pairs on neighboring chains, and radiative and nonradiative decay to the ground state. Singlet exciton annihilation and amplified spontaneous emission could be additional decay pathways. They are, however, considered negligible here, based on the relatively low excitation densities used and the similar early fluorescence dynamics in film and solution, although the excitation densities differ by orders of magnitude. As has previously been observed for structurally quite similar APFO3, ${ }^{30}$ the exciton decay in PCDTBT is biphasic. It is associated with one time constant of tens of picoseconds and one of hundreds of picoseconds. The faster of the two is mixed with spectral relaxation processes, and part of this decay thus occurs from higher-energy excitons. This is especially obvious in the film, where the spectral relaxation takes longer. The distribution of excitons with different energies due to disorder might be at the origin of the biphasic decay. Finally, there are two effects that account for the faster average exciton depopulation in the film: the relative amplitude of the fast decay component is higher in the solid state (although the time scale is similar as in solution), and the slower decay component has a much shorter time constant.

\section{Broader Relevance of the Results}

Understanding the initial relaxation processes following photoexcitation of the pristine polymer is essential to gain insight into the photoinduced charge separation mechanism that occurs in the polymer:fullerene blend. A femtosecond-resolved transient absorption study of PCDTBT: $\mathrm{PC}_{70} \mathrm{BM}$ revealed that the charged species appear faster than the $200 \mathrm{fs}$ time resolution of the instrumentation. ${ }^{27}$ The ultrafast charge transfer is particularly important for PCDTBT:PC ${ }_{70} \mathrm{BM}$. The corresponding BHJ solar cells exhibit near $100 \%$ internal quantum efficiency; nearly every photon absorbed leads to a charge separated pair of mobile carriers, and nearly all of the mobile carriers are collected at the electrodes. ${ }^{26}$ Consequently, there is no ambiguity; photoinduced charge transfer occurs with quantum efficiency approaching unity in the $100 \mathrm{fs}$ time scale.

How relevant are the relaxation processes found in the present study of pristine PCDTBT in view of this ultrafast quantitative charge separation? The answer is that all the processes that can be temporally resolved here, and which cause the observed changes in the emission spectrum of pristine PCDTBT, are irrelevant with respect to charge separation because they are at least an order of magnitude too slow. Thus, charge separation in an optimized blend occurs during (or even prior to) exciton formation, prior to conformational backbone rearrangements and certainly before exciton migration. These results therefore challenge the commonly proposed picture that, in the phaseseparated BHJ blend, diffusion of singlet excitons to an interface with a fullerene (typically a distance of about $10 \mathrm{~nm}$ ) occurs before charge separation can take place by exciton dissociation. ${ }^{6}$ As shown by the up-conversion studies reported here for PCDTBT, diffusion by exciton migration takes at least $0.5-1$ ps for a single hopping step. Thus, exciton diffusion over distances comparable to $10 \mathrm{~nm}$ cannot account for charge separation within less than 200 fs.

The same discrepancy can be generalized to many other conjugated polymers. It is well established that charge separation is ultrafast ( $\sim 100 \mathrm{fs}$ ) for highly efficient and morphologically optimized BHJ materials. ${ }^{29,33,34,86}$ In particular, for the MDMOPPV:PCBM composite, the ultrafast charge transfer (45 fs) was time-resolved. ${ }^{87}$ On the other hand, the time scale of $0.5-1 \mathrm{ps}$ for exciton hopping has also been reported in numerous publications for different polymers. ${ }^{35,39,43-46,57,58,77,78,80,81}$ Moreover, an exciton diffusion coefficient of $3 \times 10^{-3} \mathrm{~cm}^{2} / \mathrm{s}$ was found for MEH-PPV film based on studies of the exciton-exciton annihilation, ${ }^{48}$ and a one-dimensional exciton diffusion length of 5-8 nm was deduced using an exciton lifetime of $140 \mathrm{ps}$ in the pristine polymer. Assuming that this lifetime is reduced to $100 \mathrm{fs}$ in a blend with fullerene due to charge separation, the length an exciton can diffuse drops to only $0.1-0.2 \mathrm{~nm}$, clearly not sufficient to reach an interface in the BHJ.

To resolve this discrepancy, it could be argued that the charge separation is in reality much slower and occurs with a distribution of time constants that is correlated to the distribution of exciton distances from an interface. Although clearly not true for PCDTBT:PC ${ }_{70} \mathrm{BM}$, this was suggested by a visible pumpinfrared probe spectroscopy study of CN-MEH-PPV:PCBM blends, where $30 \%$ of the excitons was found to undergo charge separation in several nanoseconds because they had to diffuse over a large distance. ${ }^{88}$ Such slow components are not, however, characteristic of optimized mixtures and morphologies with high PCE and IQE values in solar cells, where all experimental evidence points to ultrafast charge separation. ${ }^{27,29,30,33,34,86}$

One could also argue that the length scale for phase separation in the BHJ of $10 \mathrm{~nm}$ is overestimated, and that the donor and acceptor are in reality so intimately mixed that every excitation in the polymer occurs so close to a fullerene that no diffusion is necessary prior to charge separation. Experimental observations have demonstrated, however, that power conversion efficiency in very intimately mixed polymer:fullerene blends is often low. As-cast films of P3HT:PCBM display intimate mixing and have a PCE of less than $1 \%{ }^{8}$ This can be increased to $5 \%$ by thermal annealing, which increases the phase separation and leads to more crystalline polymer domains with dimensions about $10 \mathrm{~nm}$. The changes in morphology that enable better PCE were clearly shown by transmission electron microscopy and X-ray diffraction data. For PBTTT, the fullerene intercalates

(86) Hwang, I.-W.; Moses, D.; Heeger, A. J. J. Phys. Chem. C 2008, 112, 4350-4354.

(87) Brabec, C. J.; Zerza, G.; Cerullo, G.; De Silvestri, S.; Luzzati, S.; Hummelen, J. C.; Sariciftci, S. Chem. Phys. Lett. 2001, 340, 232236.

(88) Barbour, L. W.; Pensack, R. D.; Hegadorn, M.; Arzhantsev, S.; Asbury, J. B. J. Phys. Chem. C 2008, 112, 3926-3934. 
into the crystalline polymer phase, but very low PCE was observed with the intimately mixed blend in spite of complete fluorescence quenching. ${ }^{89}$ Good solar cell performance for this polymer could only be achieved with a high fullerene concentration leading to phase separation and pure fullerene domains for electron transport.

Another possibility that has been suggested to account for ultrafast charge separation in annealed P3HT:PCBM blends is that exciton migration is not point-like, but that excitons are highly spatially delocalized in the ordered P3HT domains, so that they can sample distances of the order of $10 \mathrm{~nm}$ on a very fast time scale. ${ }^{90}$ To our knowledge, no experimental evidence exists that supports such a high degree of exciton delocalization (especially unlikely in amorphous polymers such as PCDTBT).

We therefore consider alternative possibilities within the general idea that the electron is transferred from the photoexcited polymer to the fullerene during intraband relaxation and before it becomes bound in a singlet exciton. This is supported by experimental results. First, charge separation occurs on a very similar time scale as the ultrafast $(\sim 100 \mathrm{fs})$ relaxation processes that lead to self-localization of the electron and hole formed by the interband $\pi-\pi^{*}$ transition. Second, our results imply relatively slow exciton formation (within about $1 \mathrm{ps}$ ), which is thus much slower than the charge separation in the polymer: fullerene blend. Third, we showed that mobile charge carriers (polarons) between neighboring polymer chains are formed before singlet exciton formation in pristine PCDTBT following $\mathrm{S}_{2}$ excitation, indicating that charge carrier generation prior to exciton formation is a viable mechanism. The mechanism we suggest for the BHJ blends does not, however, imply the formation of free polarons on neighboring polymer chains with high quantum efficiency prior to the charge separation (although interchain interactions will certainly tend to increase the carrier delocalization lengths).

The details of how photoexcitations reach the polymer: fullerene interface before exciton formation (delocalization, diffusion, drift of a mobile charge carrier, intrachain or interchain transport) are beyond the scope of this Article. We note, however, that the electrons and holes formed in the $\pi$ - and $\pi^{*}$ bands are initially both delocalized and mobile directly after the interband absorption (in contrast to excitons that are spatially localized by the Coulomb interaction and by local structural relaxation of the polymer). Thus, the primary excitations can in principle reach a fullerene interface faster than a relaxed exciton.

Assuming delocalized carriers and transport to the interface (before self-localization) by drift in a field of $10^{6} \mathrm{~V} / \mathrm{cm}$, an electron mobility of approximately $10 \mathrm{~cm}^{2} /(\mathrm{V}$ s $)$ would be required to cover $10 \mathrm{~nm}$ in $100 \mathrm{fs}$. Wave function delocalization over several repeat units would significantly decrease the distance required. Such a high mobility would be transient in nature and would exist only in the ultrafast time regime. Note that transient photoconductivity measurements in polymer: fullerene blends have demonstrated a continuing decrease of carrier mobility after photoexcitation even at significantly longer time scales. ${ }^{91}$ The mobility of nonrelaxed carriers directly after photoexcitation can therefore not be compared to steady-state

(89) Mayer, A. C.; Toney, M. F.; Scully, S. R.; Rivnay, J.; Brabec, C. J.; Scharber, M.; Koppe, M.; Heeney, M.; McCulloch, I.; McGehee, M. D. Adv. Funct. Mater. 2009, 19, 1173-1179.

(90) Marsh, R. A.; Hodgkiss, J. M.; Albert-Seifried, S.; Friend, R. H. Nano Lett. 2010, 10, 923-930.

(91) Coates, N. E.; Moses, D.; Heeger, A. J., submitted for publication. mobility measurements of structurally relaxed polarons (typically $\ll 1 \mathrm{~cm}^{2} /(\mathrm{V} \mathrm{s})$ for conjugated polymers) measured, for example, in organic field-effect transistors (OFETS) over much larger distances, where deep traps (localized states) play a larger role. Time-resolved terahertz spectroscopy revealed short-range carrier mobility in P3HT film up to $30-40 \mathrm{~cm}^{2} /(\mathrm{V} \mathrm{s}) .{ }^{92}$ In addition, quantum mechanical transport of delocalized primary excitations has been demonstrated by the experimental observation of coherent excitation energy migration within MEH-PPV chains in solution, with transport over distances sufficient to reduce polarization memory in the 50-100 fs time regime. ${ }^{93}$

In summary, we conclude that exciton diffusion is not involved in the charge transfer mechanism in polymer:fullerene BHJ blends, because it is too slow. The concepts of exciton diffusion length and exciton binding energy are therefore less important for charge separation in "plastic" solar cells than has been commonly assumed. Carrier delocalization before exciton formation with high initial mobilities and transport assisted by quantum effects offer a plausible explanation to the observation that provides the foundation for the entire field of BHJ solar cells: ultrafast photoinduced charge transfer.

\section{Conclusion}

Fluorescence up-conversion measurements allowed the elucidation of the relaxation processes occurring for PCDTBT in dilute chlorobenzene solution and solid thin films, following excitation of the first or second $\pi-\pi^{*}$ interband transition. Upon absorption in the first band with around $0.6 \mathrm{eV}$ excess energy, ultrafast relaxation processes occur within the time resolution of the experiment (100-200 fs) and lead to one-half of the Stokes shift and some loss of fluorescence polarization anisotropy. They are ascribed to the thermalization toward the band edges of electrons and holes (which are formed by photoexcitation in the conduction and valence band, respectively), and to their self-localization by local structural lattice distortions. Subsequently, the emission spectrum continues to relax to lower energy, with time constants of about $0.5 \mathrm{ps}, 5 \mathrm{ps}$, and tens of picoseconds. Those relaxation processes are accompanied by fluorescence depolarization and are attributed to the interplay of three phenomena. The final stage of exciton formation (spatial recombination and Coulomb binding of the opposite charges after relaxation to the band edges) is associated with only the subpicosecond time constant. The three time constants include successive exciton migration through localized states to lower energy due to disorder. Finally, slow conformational rearrangements of the backbone also contribute to the spectral changes and are found to be slower in the film (200 ps to completion) than in solution (100 ps to completion).

On the basis of the similar shape of the steady-state spectra and the similar emission and fluorescence anisotropy dynamics during the first $10 \mathrm{ps}$, we conclude that the conformation of the polymer chains is comparable in chlorobenzene solution and in the amorphous film cast from the same solvent. The longlived anisotropy component in solution (which is lost in the film due to interchain exciton hopping) implies local order and indicates that the chain conformation is somewhat extended. When the second absorption band is excited in either medium, there is ultrafast $(<100 \mathrm{fs})$ internal conversion from $S_{2}$ to $S_{1}$, which we were not able to resolve. Comparable relaxation

(92) Cunningham, P. D.; Hayden, L. M. J. Phys. Chem. C 2008, 112, 79287935.

(93) Collini, E.; Scholes, G. D. Science 2009, 323, 369-373. 
processes then occur within the $S_{1}$ band as with direct excitation into the $S_{1}$ band. Minor differences in the spectral relaxation are accounted for by details of the internal conversion mechanism (it leads to electrons and holes further from the band edges, which self-localize to a different distribution of bound exciton states with slower migration dynamics).

Ultrafast photoinduced charge separation is the scientific foundation of the BHJ solar cell concept. In optimized polymer: fullerene blends (including PCDTBT:PC ${ }_{70} \mathrm{BM}$ ), which are used in efficient "plastic" solar cells, the ultrafast photoinduced charge separation is a quantitative process that occurs in about $100 \mathrm{fs}$. The commonly proposed picture is that this charge separation occurs after the excitons formed by light absorption in the polymer have diffused to an interface with the fullerene component within the bulk heterojunction material. However, the data demonstrate that exciton migration is too slow to account for the observed electron transfer rate. We discuss possible explanations for this discrepancy and suggest an alternative picture for the charge transfer mechanism in BHJ blends. Here, the excitation (from a $\pi-\pi^{*}$ interband transition) reaches the polymer:fullerene interface for charge separation before it becomes spatially self-localized and bound within an exciton. Issues of exciton diffusion and exciton binding energy therefore become irrelevant to ultrafast charge transfer.

Acknowledgment. N.B. thanks the Swiss National Science Foundation for Fellowship support (fellowship for prospective researchers PBGEP2-125859). We thank the Department of Energy (BES-DOE-ER46535; A. Kini, Program Officer) for research support. We thank Konarka Technologies for providing the PCDTBT material. A.J.H. thanks Dr. Daniel Moses for stimulating discussions regarding photoinduced charge transfer in BHJ blends.

Supporting Information Available: Additional figures mentioned in the text. This material is available free of charge via the Internet at http://pubs.acs.org.

JA105290E 